\section{Various inorganic attractions}

\section{J.A. McCleverty}

Basic Inorganic Chemistry, 2nd Edn. By F. Albert Cotton, Geoffrey Wilkinson and Paul L. Gaus. Wiley: 1987. Pp.708. Hbk £34.25, \$39.90; student edn $£ 12.95, \$ 9.60$.

Mechanisms of Inorganic Reactions. By Dimitris Katakis and Gilbert Gordon. Wiley: 1987. Pp.384. £34.50, \$39.95.

Structural Methods in Inorganic Chemistry. By E.A.V. Ebsworth, David W.H. Rankin and Stephen Cradock. Blackwell Scientific: 1987. Pp.456. Hbk £29.50, $\$ 44.50 ; p b k £ 13.50$.

THESE three books have two things in common: they are all concerned with inorganic chemistry, and they all provide problems and study guides, an invaluable aid to instructors struggling to find new topics and questions for tutorials and examinations.

Basic Inorganic Chemistry is an update of an earlier text directed at those who seek a general introduction to most, but not all, aspects of the area. It is not intended to be a 'baby' version of the Advanced Inorganic Chemistry by Cotton and Wilkinson, the fifth edition of which will soon appear, but a complete text, onethird of which is dedicated to general preliminaries such as structure, bonding, solids, coordination chemistry, acids and bases, and periodicity. The middle section describes the chemistry of the 'main group' elements, by individual element or group. Part three presents transition metal, lanthanide and actinide chemistry in a style similar to the Advanced series, by horizontal divisions. The final part of the book gives outlines of special topics $\pi$-acceptor ligands and organometallic chemistry, catalytic uses of organometallics, and bioinorganic systems. Topics in solid state chemistry are barely touched on, which is a pity since this is a currently fashionable area. It is arguable, however, whether a single text in inorganic chemistry, to be manageable and useful, could cover everything of interest and possible relevance.

That minor criticism apart, this is a wellrounded book, providing a description of most of inorganic chemistry on a predominantly factual basis relating to the periodicity of the elements, but with deference to the presentation of this vast area as topics. This makes it somewhat different to and, in a sense, more historically rigorous than Huheey's book Inorganic Chemistry, for which Cotton, Wilkinson and Gaus will provide serious competition.

Katakis's and Gordon's treatment of mechanisms of inorganic reactions is not intended for specialists but more for the informed beginner, at a level requiring knowledge of basic inorganic chemistry, thermodynamics and kinetics. It reviews the relationship between mechanisms, kinetics and equilibria and touches on the important factors influencing them. There are chapters dealing with the concept of the activated complex, experimental methods, the relationship between mechanism and structure, group-, atom- and electron-transfer processes, homogeneous catalysis and inorganic photochemistry. Each section has a sensibly limited number of examples illustrating the basic principles. It is a very useful supplementary text for a course of general inorganic chemistry which illustrates mechanistic study as being a constituent part of the subject area, rather than as an activity on its own.

The book by Ebsworth, Rankin and Cradock is a joy to read and, for practitioners as well as students and instructors, is extremely valuable. It deals with the application of nuclear magnetic, quad- rupole and electron-spin resonance, rotational, vibrational, electronic and photoelectron spectroscopy to real inorganic problems. It also discusses the uses, in a structural context, of Mössbauer spectroscopy, diffraction techniques and mass spectrometry.

The jewel in this particular crown is the chapter on case histories, where real problems are confronted and the contents of the structure-solving tool box deployed to the greatest effect. I must comment that there could have been some additions, for example, on aspects of optical activity, magnetism and electrochemistry, since this is a book concerned with analysis in its widest sense. These areas have as much to do with structure as, say, mass spectrometry, and are all part of the serious inorganic researcher's structure-solving kit. This is not a criticism - merely an expression of greed for more of this excellent repast.

J. A. McCleverty is a Professor of Inorganic Chemistry in the Department of Chemistry, University of Birmigham, PO Box 363, Birmingham B15 2TT, UK.

\section{Technical merit}

\section{T. S. West}

Instrumental Analysis in the Biological Sciences. By M. H. Gordon and R. Macrae. Blackie: 1987. Pp.244. Pbk£17.95.

Introduction to Instrumental Analysis. By Robert D. Braun. McGraw-Hill: 1987. Pp.1,004. \$52.95, £45.95; student edn $£ 14.95$.

IN RECENT years chemical analysis has undergone a revolutionary change which, although familiar to the chemists and physical scientists who have been responsible for it, is less well known to biological scientists. Biologists, however, can gain enormously from these new, and generally highly sophisticated, instrumental techniques, many of which employ microprocessors to simplify and improve their operation and make them more userfriendly.

Gordon and Macrae's informative and well-balanced book succeeds admirably in providing an easy-to-assimilate account of the standard separation-determination techniques, such as liquid-phase chromatography, gas-liquid chromatography and electrophoresis, that have become indispensable in the biological laboratory. Spectroscopic techniques, which are the most widely used of all physico-chemical investigative procedures, are considered in categories such as ultraviolet and visible spectrometry, fluorescence and phosphorescence, and infrared absorption spectrometry. Nuclear magnetic resonance, electron spin resonance and elec- tron paramagnetic resonance spectrometries are dealt with well, but in less detail than is probably warranted by their everincreasing importance in biology. The treatment of flame emission, atomic absorption and atomic fluorescence spectrometries is thorough enough for the intended readership, even perhaps too much so, but the electrothermal atomization techniques deserved more attention in relation to their applicability to biological materials. The sections on organic mass spectrometry and electrochemical devices are brief but useful and largely to the point.

The authors could profitably have devoted more attention to the nature of the structural information that can be won by many of the techniques they consider. They might also have addressed the problems of correct sampling of biological tissues, without which all the sophisticated science may be pointless. But, in all, this is a most useful introduction to the theory and general instrumental background of modern physico-chemical investigative techniques.

Robert Braun has produced a much larger book, with different aims. At the least, he deserves full marks for effort in compiling such a detailed - sometimes almost laborious - review of modern instrumental techniques.

Like most comprehensive texts, this one has its good and its less good points. For me the introductory treatment of electronic circuitry, operational amplifiers, logic devices and microcomputers in instrumental 'innards' is very worthwhile, and the inclusion of photoacoustic spectrometry, refractometry, thermo- 